\title{
Influence of scanning and reconstruction parameters on quality of three-dimensional surface models of the dental arches from cone beam computed tomography
}

\author{
Bassam Hassan • Paulo Couto Souza • \\ Reinhilde Jacobs • Soraya de Azambuja Berti • \\ Paul van der Stelt
}

Received: 16 December 2008 / Accepted: 18 May 2009/Published online: 9 June 2009

(C) The Author(s) 2009. This article is published with open access at Springerlink.com

\begin{abstract}
The study aim is to investigate the influence of scan field, mouth opening, voxel size, and segmentation threshold selections on the quality of the three-dimensional (3D) surface models of the dental arches from cone beam computed tomography (CBCT). 3D models of 25 patients scanned with one image intensifier CBCT system (NewTom 3G, QR SLR, Verona, Italy) using three field sizes in openand closed-mouth positions were created at different voxel size resolutions. Two observers assessed the quality of the models independently on a five-point scale using specified criteria. The results indicate that large-field selection reduced the visibility of the teeth and the interproximal space. Also, large voxel size reduced the visibility of the occlusal surfaces and bone in the anterior region in both maxilla and mandible. Segmentation threshold was more variable in the maxilla than in the mandible. Closed-mouth scan complicated separating the jaws and reduced teeth surfaces visibility. The preliminary results from this imageintensifier system indicate that the use of medium or small scan fields in an open-mouth position with a small voxel is recommended to optimize quality of the 3D surface model
\end{abstract}

\footnotetext{
B. Hassan $(\bowtie) \cdot$ P. van der Stelt

Department of Oral Radiology,

Academic Centre for Dentistry Amsterdam (ACTA),

Louwesweg 1,

1066EA Amsterdam, The Netherlands

e-mail: b.hassan@acta.nl

URL: www.acta.nl

P. Couto Souza $\cdot$ R. Jacobs $\cdot$ S. de Azambuja Berti

Oral Imaging Centre, School of Dentistry,

Department of Oral Pathology and Maxillofacial Surgery,

Faculty of Medicine, Catholic University Leuven,

Kapucijnenvoer 7 ,

3000 Leuven, Belgium
}

reconstructions of the dental arches from CBCT. More research is needed to validate the results with other flatpanel detector-based CBCT systems.

Keywords Cone beam computed tomography .

Three-dimension models · Image quality · Dentistry ·

Surface rendering

\section{Introduction}

Computerized three-dimensional (3D) models of the craniofacial region are a recent trend in dentistry. 3D models of the hard-tissue dental arches including the teeth and the jawbones have been used for various clinical applications including pre-operative treatment planning of dental implants and craniofacial surgical procedures, fabrication of dental and craniofacial prosthesis, analysis of arch dimensions, virtual treatment simulation in orthodontics, and postoperative treatment outcome assessment [1-6]. 3D models of the dental arches are typically obtained by digitizing the dental cast with high-resolution surface laser scanning [7-10]. Those models can be used to evaluate the dentition and inter-occlusal space; however, they do not provide information regarding the amount of alveolar bone available and the relation of the tooth root to its socket. In addition, the accuracy of the digitized model is limited by the accuracy of the dental impression and cast, which could be variable and inconsistent overtime depending on several factors [11-13].

3D models of the dental arches can also be obtained from computed tomography (CT) scans. Conventional CT technology provides accurate reconstructions of the alveolar bone but not the teeth due to limited spatial resolution 
and strong streak artifacts caused by metallic dental restorations and orthodontic brackets when present. Therefore, "composite models" were developed where the highquality $3 \mathrm{D}$ reconstructions of the alveolar bone and the roots of the teeth obtained from $\mathrm{CT}$ were combined with the high-quality $3 \mathrm{D}$ reconstructions of the teeth crowns obtained from laser surface scanning of the dental cast. And, while this technique produced satisfactory results, it was deemed unsuitable for the clinical practice due to time and cost constraints associated with complicated setup and the extensive user experience required for correct implementation [2, 14-20].

Cone beam CT (CBCT) specifically developed for the maxillofacial region provides comparable images to conventional $\mathrm{CT}$ at reduced radiation dose and cost [21-26]. CBCT reconstructions at present have smaller voxels in comparison with conventional CT [27], which could be advantageous in obtaining more accurate $3 \mathrm{D}$ surface models of the teeth crowns. However, CBCT subjective image quality is still inferior in comparison with conventional CT. Several artifacts including beam hardening and inhomogeneity and truncation influence image contrast and bone border definition in CBCT [28-31]. The Feldkamp filtered back-projection reconstruction algorithm employed by most CBCT manufacturers is exactly identical to the Radon inverse-transform algorithm used in conventional $\mathrm{CT}$ in the mid-plane [32]. As such, it does not inherently reduce the effect of streak artifacts caused by metallic dental filling and orthodontic brackets. Moreover, since CBCT is inferior to CT in terms of contrast resolution, the effect of metal streak artifacts may be even more pronounced [33]. Several reconstruction algorithms and techniques were proposed to reduce those artifacts in CBCT images, but they remain to date computationally expensive and not yet widely adopted by CBCT manufacturers [34].

There are currently many $\mathrm{CBCT}$ systems which are commercially available. Those systems are categorized according to detector design technology into: (1) image intensifier tube/charged coupled devices (IIT/CCD) combination or (2) flat panel detector (FPD) [34]. It has been reported that IIT/CCD suffers from more artifacts and increased noise levels compared to FPD systems [35]. Also, in practice several patient scanning and data reconstruction parameters have influence on CBCT subjective image quality $[35,36]$. All those factors combined could thus directly influence the quality of the 3D surface models reconstructions of the dental arches from CBCT. It is necessary to assess the quality of those models and the influence those parameters might have in order to optimize patient scanning, data reconstruction, and 3D surface model creation protocols. This is important in order to assess whether it is possible to obtain accurate 3D surface models of the dental arches from CBCT or not. Few studies based on dry skull samples assessed the accuracy of 3D models reconstructions from $\mathrm{CBCT}[37,38]$. However, 3D surface models created from phantoms, dry skulls, or even formalin-fixed cadavers are not realistic and do not represent actual patient. The quality of the segmented model could deviate largely from what is observed clinically. The objective of this study is to assess the quality of 3D surface models of the dental arches with respect to the influence of different scanning and reconstruction parameters in one IIT-based CBCT system (NewTom $3 \mathrm{G}$ ) in an in vivo sample of patients.

\section{Materials and methods}

Sample selection

Twenty-five datasets scanned with the IIT/CCD system Newtom 3G CBCT (QR SLR, Verona, Italy) were selected from a larger database of CBCT patients. The datasets were divided into three groups: Group (A) consisted of ten patients scanned with the 12-in. (large) detector scan field of view (FoV). Group (B) consisted of ten patients scanned with the 9-in. (medium) scan FoV. Group (C) consisted of five patients scanned with the 6-in. (small) scan FoV. Groups (A) and (B) were scanned in a closed-mouth position with the teeth in maximum intercuspid relation, while group (C) was scanned in an open-mouth position. Additional selection criteria were that (1) the upper and the lower jaws are both visible in the scan, (2) no more than four teeth are missing for both jaws excluding the third molars, and that (3) there are no orthodontic brackets or large metal restorations. Informed consent was obtained from the patients to use their data for research purposes. Datasets were exported according to the manufacturer's default settings for each scan field in DICOM 3 file format at the isotropic voxel size of $\left(0.3,0.25\right.$, and $\left.0.2 \mathrm{~mm}^{3}\right)$ for the 12-, 9-, and 6-in. scan FoVs, respectively. The datasets were 12 bits in depth, and the gray values $\left(2^{12}=4,096\right)$ range was $(-1,000$ to 3,095$)$. The datasets were imported into 3D analysis software (Amira v4.2, Visage Imaging, Carlsbad, CA, USA) for making the 3D models.

Creating the 3D models

\section{Segmentation threshold}

The histogram of a CBCT dataset is composed of a wide range of gray-scale values that represent the X-ray attenuation profiles of the different soft and hard tissues. It is generally more difficult to specify the correct threshold value to separate the bone from soft tissue and background in $\mathrm{CBCT}$ than in conventional $\mathrm{CT}$ due to inherent 
inconsistencies in the histogram. A single threshold value was specified to segment the bone and teeth from the background and soft tissue for each 3D model. The surface models were created using the marching cube algorithm [39]. To determine an optimal threshold value, the histogram of each model was approximated as a mixture of Gaussians by using the stochastic expectation maximization (SEM) algorithm. The threshold value was then specified in the region of the intersection of the two Gaussians representing soft tissue and bone. To eliminate the stochastic part of the SEM, the average of three threshold values was taken [29, 40]. Threshold values were determined using this method separately for the maxilla and for the mandible when the jaws were separated (Fig. 1).

\section{Scan field selection}

For groups (A), (B), and (C), a smaller region of interest (ROI) limited only to the dental arches was selected, and the rest was digitally removed (Fig. 2). In each group, the upper arch was separated from the lower arch by using a cubic ROI selection. For this experiment, the isotropic voxel size of $0.3 \mathrm{~mm}^{3}$ was chosen for each model. Fifty $3 \mathrm{D}$ surface models were created in total (25 upper and 25 lower arches) for the three groups (A, B, C).

\section{Voxel size selection}

Group (C) was selected to assess the influence of the voxel size on $3 \mathrm{D}$ model quality. This was done in order to assess the influence of voxel size selection on the definition of the occlusal surfaces since groups (A) and (B) were scanned in closed-mouth position that the occlusal surfaces were difficult to visualize. To assess the influence of each level of the "voxel size" factor independently, voxels were categorized into two components: (a) pixel resolution in the $(x, y)$ scan plane (PRxy) and (b) axial slice thickness (AST) in the $(z)$ plane. First, PRxy was fixed at $0.3 \mathrm{~mm}$, and AST was increased from 0.3 to $1.2 \mathrm{~mm}$ using $0.3 \mathrm{~mm}$ steps. Then, AST was fixed at $0.3 \mathrm{~mm}$ and PRxy was increased from 0.3 to $1.2 \mathrm{~mm}$ using $0.3 \mathrm{~mm}$ steps. A 3D bicubic resampling filter was used to manipulate the voxel size to change the pixel resolution and the axial slice thickness. The bi-cubic resampling filter uses information from 16 adjacent points to each voxel for resampling the data. This has the added advantage of smoother resampling and less interpolation artifact compared to bilinear or the nearest neighbor filtering. In total, eight voxel combinations were obtained per model (AST 0.3, 0.6, 0.9, 1.2 and PRxy $0.3,0.6,0.9,1.2)$. A total of 80 models were created (40 upper and 40 lower) dental arches.

\section{Observations}

A single investigator who did not participate in the observations created the models. All 3D models were coded, and two observers (one maxillofacial radiologist and one maxillofacial surgeon) assessed the quality of all the models independently. The observers were blind to the scan field and voxel size selections. The models were
Fig. 1 An example of the segmentation procedure and threshold value determination for a 3D model from group (C) in $3 \mathrm{D}(\mathbf{a}, \mathbf{c})$ and on 2D axial slices $(\mathbf{b}, \mathbf{d})$. The model can have an increased noise level due to threshold underestimation (a and b) or several holes in the bone and teeth due to threshold overestimation (c and d)
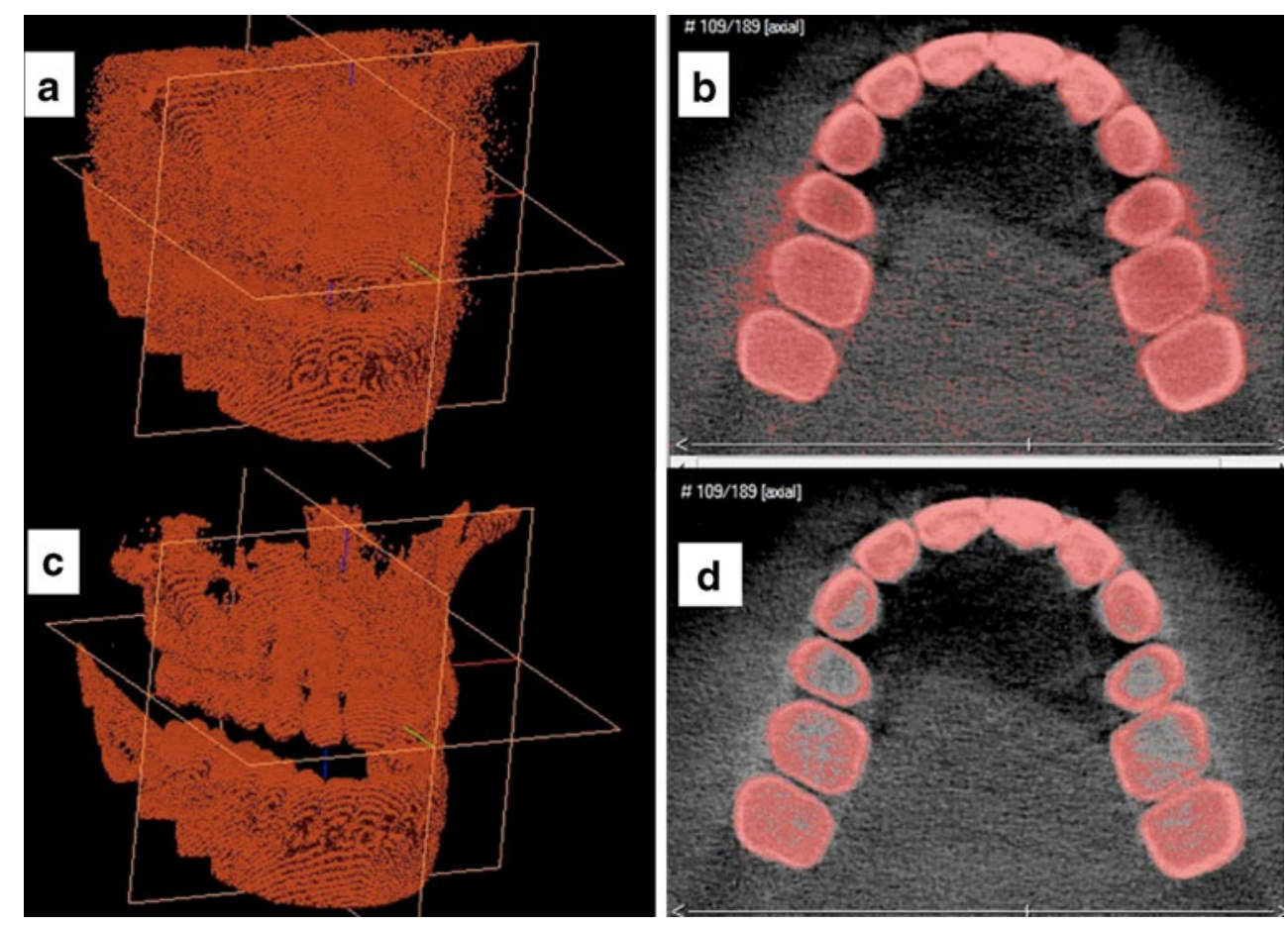
Fig. 2 A dataset from group (A) large scan field. Example of limiting the selection to the dental arches by using a cubic region of interest. The original dataset (left) and the cropped model (right)

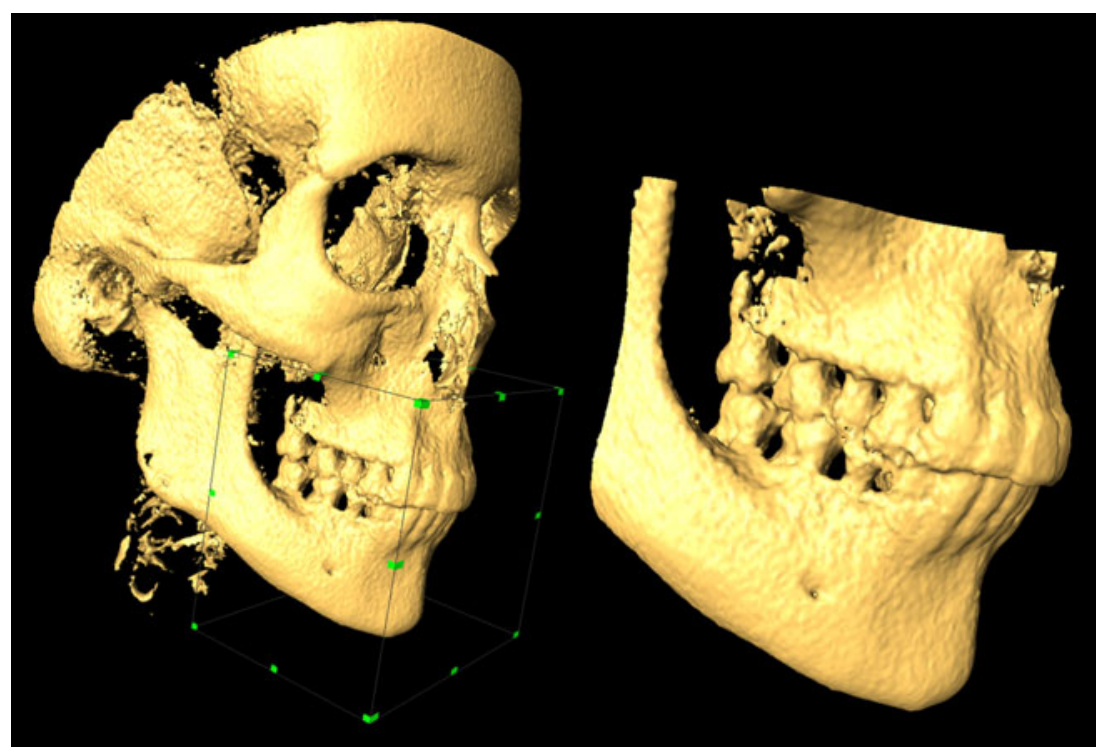

presented to the observers in a random order. All models were viewed on a 19-in. flat panel screen $(1,280 \times 1,024$, Philips Brilliance 200WP, Brussels, Belgium). The observers were allowed to rotate, scale (zoom), and translate (move) the models to improve visibility of certain structures, but adjusting the threshold value was not permitted. The observers were asked to assess the subjective visibility of the following structures for each model in the maxilla and the mandible on a five-point scale $(1=$ very poor, $2=$ poor, 3 = satisfactory, $4=$ good, 5 = very good):

1. External surfaces of the teeth and the occlusal surfaces in group $(\mathrm{C})$

2. Alveolar bone anteriorly and posteriorly

3. Palate region

4. Interproximal space between the teeth anteriorly and posteriorly separately

5. Overall image noise which represented the "soft tissue" and background noise that routinely appears in most CBCT surface reconstructions due to the difficulty in specifying a single threshold value to segment bone

\section{Statistical analysis}

The observation data were entered and analyzed using SPSS software (SPSS v.15, SPSS, Chicago, IL, USA). The observers' ratings were entered into a multivariate analysis of variance (MANOVA) with the Wilk's lambda test to assess the influence of scan field, voxel size, and segmentation threshold on visibility of the different structures of the 3D models. The three scan field groups and the different voxel combinations were compared to each other using Helmert planned contrasts. Inter-observer agreement was determined using Cohen kappa, and observers' interaction with scan FoV selection was calculated in the MANOVA analysis. Alpha level was set to 0.05

\section{Results}

Segmentation threshold

Mean threshold values were $(660 \pm 44.72,850 \pm 51.63$, and $784 \pm 60.82$ ) for groups (A), (B), and (C), respectively. There was a statistically significant difference in the threshold value among the three groups $(p=0.001)$. Mean threshold and standard deviation value were (847 \pm $33.21)$ and $(662 \pm 121.2)$ for the mandible and maxilla, respectively.

Field size selection

There was an overall statistically significant difference among the three groups (A, B, and C) in 3D surface model quality $(p=0.0001)$. Inter-observer agreement was moderate (kappa $=0.53$ ). There was no statistically significant interaction between the observers and the scan field groups $(p=0.12)$. Specifically, the visibility of the external surfaces of the teeth in the maxilla and the interproximal space between the teeth in the anterior region in the maxilla and the mandible was better in group $(C)$ than in group (A) ( $p=0.0001)$ with no significant differences from group (B) ( $p=0.48 ;$ Fig. 3$)$. Image noise was significantly 

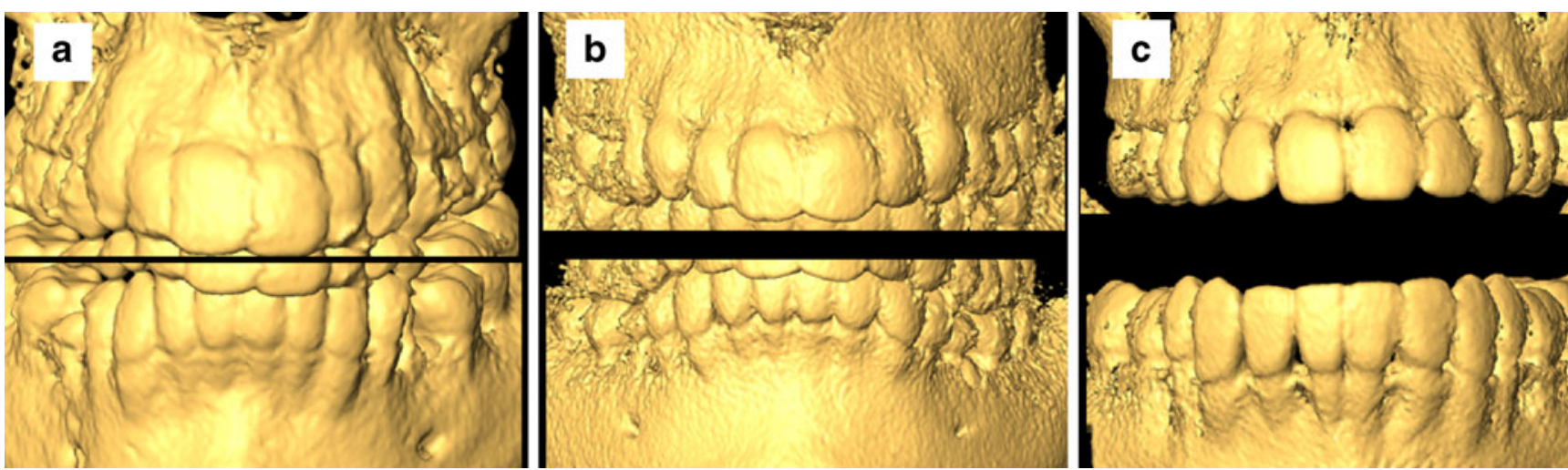

Fig. 3 Influence of scan field selection on the quality of the 3D model reconstruction. The difference in the visibility of the teeth and interproximal space between a large, $\mathbf{b}$ medium, and $\mathbf{c}$ small fields

less in group (A) than in groups (B) and (C) $(p=0.0001$, $0.001)$, respectively.

Voxel size selection

There was an overall statistically significant difference in $3 \mathrm{D}$ model quality in group $(\mathrm{C})$ due to manipulating pixel resolution in the scan plane (PRxy; $p=0.0001)$ but not due to axial slice thickness manipulation $(p=0.87)$. There was no significant interaction between the observers and PRxy $(p=0.08)$. Specifically, the visibility of the external and occlusal surfaces of the teeth, anterior alveolar bone, and interproximal space anteriorly in the maxilla and the mandible was significantly improved by the small pixel resolution $\mathrm{PRxy}=0.3 \mathrm{~mm}$ in comparison with other selections ( $p=0.001$; Fig. 4). There were no significant differences in subjective image quality between $(\mathrm{PRxy}=$ $0.6 \mathrm{~mm})$ and $(\mathrm{PRxy}=0.9 \mathrm{~mm})$ selections $(p=0.17)$ but a significant difference between $(\mathrm{PRxy}=0.6 \mathrm{~mm})$ and $(\mathrm{PRxy}=$ $1.2 \mathrm{~mm})$ selections $(p=0.03)$.

\section{Discussion}

The results indicate that scan field selection has significant influence on the quality of the 3D models. The small scan field selection provided best visibility for the different structures of the 3D models. Interestingly, there was no significant difference between the small and the medium scan fields in the quality of the 3D models. This means that the medium field, which covers the temporomandibular joints bilaterally and several other important anatomical regions including the maxillary sinus, can be used without loss in quality. The large field reduced the visibility of the teeth surfaces and the interproximal space between the teeth. This is significant because the standard scanning
Fig. 4 Influence of voxel size manipulation on the quality of the $3 \mathrm{D}$ model reconstruction. Changing the pixel resolution in the scan plane PRxy influences the visibility of the occlusal surfaces of the teeth between a $0.3 \mathrm{~mm}, \mathbf{b} 0.6 \mathrm{~mm}, \mathbf{c} 0.9 \mathrm{~mm}$, and $\mathbf{d} 1.2 \mathrm{~mm}$. Notice the decreased noise level associated with large voxel on $\mathbf{d}$ in comparison with a
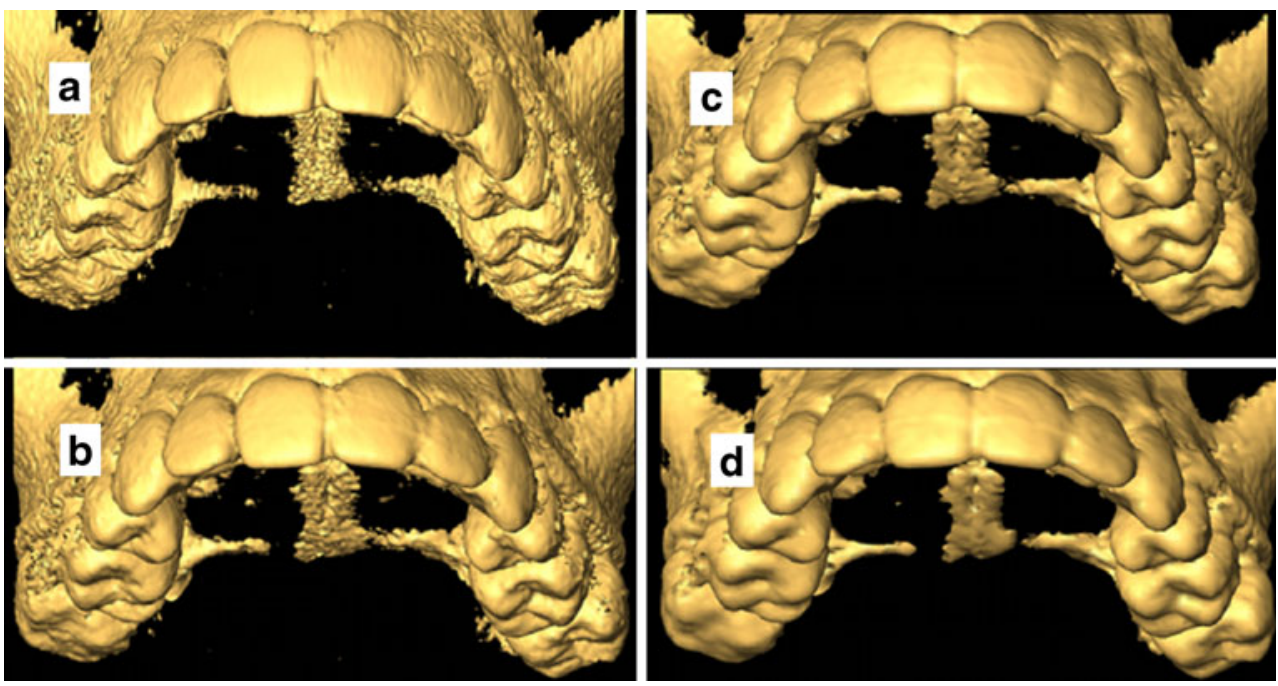
protocol of orthodontic patients with $\mathrm{CBCT}$ is with the large scan field. The results here show, however, that there is a significant loss in quality of the $3 \mathrm{D}$ models of the dental arches when the large field is used. Conversely, there were more image artifacts associated with the small scan field than with the large scan field. This could be due to cone truncation for small field selections.

An important factor when comparing different scan FoVs is the local tomography problem. When a small FoV is selected, the anatomical structures outside the region of interest are imaged as well since the line integrals sampled by the detector pass through them. Due to the fact that those tissues are only sampled for a small angular range, the resulting image reconstruction is inconsistent [41-43]. Smaller CBCT scan FoVs suffer from greater variability in the density gray values as well (i.e., more inconsistencies) compared to larger scan FoVs [35, 44].

The different scan fields have different voxel sizes, and various CBCT scanners also differ from each other in voxel size selections for each scan field. The importance of voxel size stems from a practical observation that very small voxels (e.g., 0.2 or $0.3 \mathrm{~mm}^{3}$ isotropic) result in an extremely large surface mesh model, which is difficult to process to create an accurate $3 \mathrm{D}$ surface model for preoperative treatment planning and simulation. The results here show that large pixel resolution of $0.6,0.9$, or $1.2 \mathrm{~mm}$ in the $x, y$ plane significantly reduce the visibility of the occlusal surfaces of the teeth, interproximal space between the teeth, and alveolar bone. Interestingly, increasing the axial slice thickness in the $z$ plane up to $0.9 \mathrm{~mm}$ did not significantly reduce the quality of the $3 \mathrm{D}$ models. That means that anisotropic voxels with small pixel area in the $x, y$ plane and larger slice thickness can be used instead of the "standard" small isotropic voxels. This has the added advantage of significantly reducing the model size to facilitate processing while maintaining image quality and also to reduce image noise. The choice of larger voxels reduces image noise, which is caused by photon count statistics, by averaging the gray-level values across slices, which in turn, reduces the overall noise level in the image [45].

Segmentation threshold value was automatically determined and observer independent [29]. There was more variation in the threshold value due to scan field selection than due to the different samples within the same field. Variation in the threshold value was less in the mandible than in the maxilla. This can be explained that cortical bone in the mandible is thick enough to keep the attenuation profile uniform across the entire bone surface, while in the maxilla, the varied thin cortical bone especially in the palate and tuberosity regions creates significant "bone dehiscence and fenestration" artifacts effect in the 3D model. Due to limited contrast, the roots could not be separated from their sockets using binary thresholding alone without creating artifacts. No attempt was made to systematically evaluate the visibility of the roots since they were very difficult to visualize.

A closed-mouth position made it extremely difficult to separate the jaws using a cubic ROI selection to visualize the occlusal surfaces of the teeth and to assess the interocclusal relationship. A closed-mouth position necessitates manual segmentation to separate the teeth from each other, which was a tedious, time-consuming, and user-dependent procedure that resulted in unsatisfactory results. An openmouth position facilitates separating the teeth to visualize the interproximal space. The mandible can be then virtually rotated into maximum occlusion using software tools.

This study was conducted to evaluate the influence of several scanning and reconstruction parameters on the quality of 3D model reconstructions of the dental arches from one IIT/CCD CBCT scanner (NewTom 3G). The required surface description includes clear display of the teeth surfaces and the occlusal contacts, exact reconstruction of the alveolar bone, plus separation of the teeth from each other and each tooth root from its socket. The study results show that it is still difficult to meet all those demands since separating the root from its socket is still difficult to achieve. Also, to produce a "usable" 3D surface model with sufficient quality, several scanning and reconstruction parameters need to be optimized first, and an optimized segmentation approach must be adhered to.

This study was limited in that no interaction between the scan field selection and the voxel size was assumed. For practical time and resources constraints, it was not possible to assess the influence of voxel size selection on all fields. Instead, the small field was selected a priori based on the hypothesis that it provides better subjective image quality. That hypothesis was supported by the study results. Also, the visibility of the occlusal surfaces with the large and

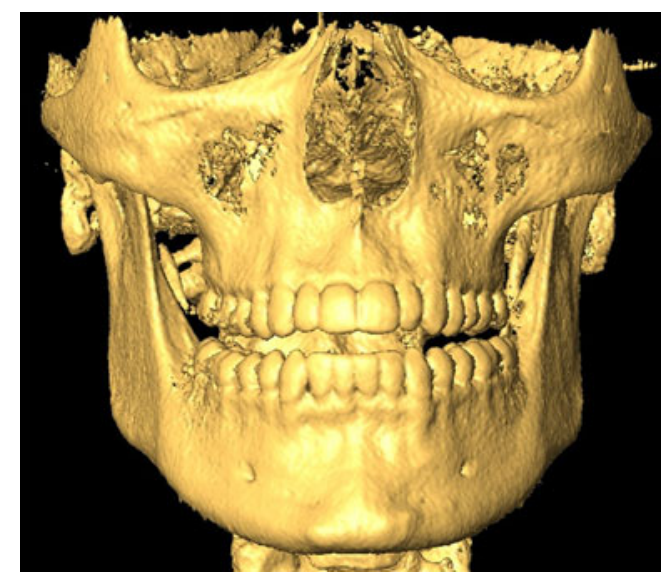

Fig. 5 Example of the recommended optimized scan protocol for making 3D reconstructions of the dental arches from CBCT. Medium field, open-mouth position with an anisotropic voxel of $0.4 \times 0.6 \mathrm{~mm}$ 
medium fields were not assessed since it was not possible to obtain scan material with the 9- and the 12-in. scan fields with open-mouth position. The study is limited only to one IIT/CCD CBCT system (NewTom 3G). Other FPD based systems are different with respect to image quality and scan and reconstruction parameters. Therefore, caution should be exercised when extrapolating the results to other $\mathrm{CBCT}$ systems. Lastly, the influence of streak artifacts was not investigated to limit the number of assessed factors on subjective image quality. However, it is expected that streak artifacts will have a significant influence on the quality of the 3D models.

In conclusion, within the limitations of the current study, several scanning and reconstruction parameters need to be optimized first before good-quality models can be created. The use of the small or medium field in an open-mouth scan position coupled with an anisotropic voxel of $0.3-0.4 \mathrm{~mm}$ pixel resolution in the scan plane and an axial slice thickness of $0.6-0.7 \mathrm{~mm}$ is recommended when creating $3 \mathrm{D}$ models of the dental arches from NewTom 3G CBCT (Fig. 5).

Conflict of interest The authors declare that they have no conflict of interest.

Open Access This article is distributed under the terms of the Creative Commons Attribution Noncommercial License which permits any noncommercial use, distribution, and reproduction in any medium, provided the original author(s) and source are credited.

\section{References}

1. Cevidanes LH, Bailey LJ, Tucker SF et al (2007) Threedimensional cone-beam computed tomography for assessment of mandibular changes after orthognathic surgery. Am J Orthod Dentofacial Orthop 131:44-50

2. Caloss R, Atkins K, Stella JP (2007) Three-dimensional imaging for virtual assessment and treatment simulation in orthognathic surgery. Oral Maxillofac Surg Clin North Am 19:287-309

3. Van Steenberghe D, Malevez C, Van Cleynenbreugel J et al (2003) Accuracy of drilling guides for transfer from threedimensional CT-based planning to placement of zygoma implants in human cadavers. Clin Oral Implants Res 14:131-136

4. Van Assche N, van Steenberghe D, Guerrero ME et al (2007) Accuracy of implant placement based on pre-surgical planning of three-dimensional cone-beam images: a pilot study. J Clin Periodontol 34:816-821

5. Jacobs R, Adriansens A, Verstreken K (1999) Predictability of a three-dimensional planning system for oral implant surgery. Dento-maxillo-facial Radiol 28:105-111

6. Cevidanes LHS, Bailey LJ, Tucker GR et al (2005) Superimposition of 3D cone-beam CT models of orthognathic surgery patients. Dento-maxillo-facial Radiol 34:369-375

7. Wang Y, Zhao Y, Lv P et al (2006) A scanning method for the dental casts of intercuspal position. Beijing Da Xue Xue Bao 18 (38):298-300

8. Lu P, Li Z, Wang Y (1999) A study of dental cast by using 3D laser non-contact measurement and analysis. Zhonghua Kou Qiang Yi Xue Za Zhi 34:351-353
9. Da Silveira AC, Daw JL, Kusnoto B et al (2003) Craniofacial applications of three-dimensional laser surface scanning. J Craniofac Surg 14:449-456

10. Lu P, Li Z, Wang Y et al (2000) The research and development of noncontact 3-D laser dental model measuring and analyzing system. Chin J Dent Res 3:7-14

11. DeLong R, Heinzen M, Hodges JS et al (2003) Accuracy of a system for creating 3D computer models of dental arches. J Dent Res 82:438-442

12. Petrie CS, Walker MP, O'mahony AM et al (2003) Dimensional accuracy and surface detail reproduction of two hydrophilic vinyl polysiloxane impression materials tested under dry, moist, and wet conditions. J Prosthet Dent 90:365-372

13. DeLong R, Pintado MR, Ko CC et al (2001) Factors influencing optical 3D scanning of vinyl polysiloxane impression materials. J Prosthodont 10:78-85

14. Gateno J, Xia J, Teichgraeber JF et al (2003) A new technique for the creation of a computerized composite skull model. J Oral Maxillofac Surg 61:222-227

15. Uechi J, Okayama M, Shibata T et al (2006) A novel method for the 3-dimensional simulation of orthognathic surgery by using a multimodal image-fusion technique. Am J Orthod Dentofacial Orthop 130:786-798

16. Swennen GRJ, Barth E, Eulzer C et al (2007) The use of a new 3D splint and double CT scan procedure to obtain an accurate anatomic virtual augmented model of the skull. Int $\mathrm{J}$ Oral Maxillofac Surg 36:146-152

17. Swennen GRJ, Mommaerts MY, Abeloos J et al (2007) The use of a wax bite wafer and a double computed tomography scan procedure to obtain a three-dimensional augmented virtual skull model. J Craniofac Surg 18:533-539

18. Nkenke E, Zachow S, Benz M et al (2004) Fusion of computed tomography data and optical 3D images of the dentition for streak artefact correction in the simulation of orthognathic surgery. Dento-maxillo-facial Radiol 33:226-232

19. Metzger MC, Hohlweg-Majert B, Schwarz U et al (2008) Manufacturing splints for orthognathic surgery using a threedimensional printer. Oral Surg Oral Med Oral Pathol Oral Radiol Endod 105:e1-e7

20. Nkenke E, Vairaktaris E, Neukam FW et al (2007) State of the art of fusion of computed tomography data and optical 3D images. Int J Comput Dent 10:11-24

21. Quereshy FA, Savell TA, Palomo JM (2008) Applications of cone beam computed tomography in the practice of oral and maxillofacial surgery. J Oral Maxillofac Surg 66:791-796

22. Loubele M, Bogaerts R, Van Dijck E, et al (2008) Comparison between effective radiation dose of CBCT and MSCT scanners for dentomaxillofacial applications. Eur J Radiol (July 2008; in press)

23. Silva MAG, Wolf U, Heinicke F et al (2008) Cone-beam computed tomography for routine orthodontic treatment planning: a radiation dose evaluation. Am J Orthod Dentofacial Orthop 133 (640):e1-e5

24. Araki K, Maki K, Seki K et al (2004) Characteristics of a newly developed dentomaxillofacial X-ray cone beam CT scanner (CB MercuRay): system configuration and physical properties. Dentomaxillo-facial Radiol 33:51-59

25. Sukovic P (2003) Cone beam computed tomography in craniofacial imaging. Orthod Craniofac Res 6(Suppl):131-136 discussion 179-82

26. Yajima A, Otonari-Yamamoto M, Sano T et al (2006) Cone-beam CT (CB Throne) applied to dentomaxillofacial region. Bull Tokyo Dent Coll 47:133-141

27. Bartling SH, Majdani O, Gupta R et al (2007) Large scan field, high spatial resolution flat-panel detector based volumetric CT of the whole human skull base and for maxillofacial imaging. Dentomaxillo-facial Radiol 36:317-327 
28. Loubele M, Guerrero ME, Jacobs R et al (2007) A comparison of jaw dimensional and quality assessments of bone characteristics with cone-beam CT, spiral tomography, and multi-slice spiral CT. Int J Oral Maxillofac Implants 22:446-454

29. Loubele M, Maes F, Schutyser F et al (2006) Assessment of bone segmentation quality of cone-beam CT versus multislice spiral CT: a pilot study. Oral Surg Oral Med Oral Pathol Oral Radiol Endod 102:225-234

30. Loubele M, Maes F, Jacobs R et al (2008) Comparative study of image quality for MSCT and CBCT scanners for dentomaxillofacial radiology applications. Radiat Prot Dosimetry 129:222-6

31. Loubele M, Jacobs R, Maes F et al (2008) Image quality vs radiation dose of four cone beam computed tomography scanners. Dento-maxillo-facial Radiol 37:309-319

32. Feldkamp LA, Davis LC, Kress JW (1984) Practical cone-beam algorithm. J Opt Soc Am A 1:612-619

33. Zhang Y, Zhang L, Zhu XR et al (2007) Reducing metal artifacts in cone-beam CT images by preprocessing projection data. Int $\mathrm{J}$ Radiat Oncol Biol Phys 1(67):924-932

34. Scarfe WC, Farman AG (2008) What is cone-beam CT and how does it work? Dent Clin North Am 52(4):707-730

35. Katsumata A, Hirukawa A, Okumura S, Naitoh M, Fujishita M, Ariji E, Langlais RP (2007) Effects of image artifacts on grayvalue density in limited-volume cone-beam computerized tomography. Oral Surg Oral Med Oral Pathol Oral Radiol Endod 104 (6):829-836

36. Kwong JC, Palomo JM, Landers MA et al (2008) Image quality produced by different cone-beam computed tomography settings. Am J Orthod Dentofacial Orthop 133:317-327

37. Periago DR, Scarfe WC, Moshiri M, Scheetz JP, Silveira AM, Farman AG (2008) Linear accuracy and reliability of cone beam
CT derived 3-dimensional images constructed using an orthodontic volumetric rendering program. Angle Orthod 78(3):387395

38. Hassan B, van der Stelt P, Sanderink G (2009) Accuracy of threedimensional measurements obtained from cone beam computed tomography surface-rendered images for cephalometric analysis: influence of patient scanning position. Eur J Orthod 31(2):129134

39. Lorensen WE, Cline HE (1987) Marching cubes: a high resolution 3D surface construction algorithm. SIGGRAPH Comput. Graph 21:163-169

40. Baillard C, Barillot C, Bouthemy P (2000) Robust adaptive segmentation of 3-D medical images with level sets. INRIA 4071:1-26

41. Siltanen S, Kolehmainen V, Järvenpää S, Kaipio JP, Koistinen $P$, Lassas M, Pirttilä J, Somersalo E (2003) Statistical inversion for medical x-ray tomography with few radiographs: I. General theory. Phys Med Biol 48(10):1437-1463

42. Kuchment P, Lancaster K, Mogilevskaya L (1995) On local tomography. Inverse Probl 11(3):571-589

43. Katsevich AI (1997) Local tomography for the limited-angle problem. J Math Anal Appl 213:160-182

44. Katsumata A, Hirukawa A, Okumura S, Naitoh M, Fujishita M, Ariji E, Langlais RP (2009) Relationship between density variability and imaging volume size in cone-beam computerized tomographic scanning of the maxillofacial region: an in vitro study. Oral Surg Oral Med Oral Pathol Oral Radiol Endod 107 (3):420-425

45. Salvado O, Hillenbrand CM, Wilson DL (2006) Partial volume reduction by interpolation with reverse diffusion. Int $\mathrm{J}$ Biomed Imaging Volume 2006:920-922 